\title{
HARMONIC ANALYSIS ON TORIC VARIETIES
}

\author{
BERNARD SHIFFMAN, TATSUYA TATE, AND STEVE ZELDITCH
}

\begin{abstract}
Harmonic analysis on a toric Kähler variety $(M, \omega)$ refers to the orthonormal basis of eigenfunctions of the $\left(\mathbb{C}^{*}\right)^{m}$ action on the spaces $H^{0}\left(M, L^{N}\right)$ of holomorphic sections of powers of the positive line bundle $L \rightarrow M$ with $c_{1}(L)=[\omega]$, and the Fourier multipliers that act on them. Using this harmonic analysis, we give an exact formula for the Szegö kernel as a Fourier multiplier $\mathcal{M}$ applied to the pull back of the Szegö kernel of projective space under a monomial embedding. The Fourier multiplier $\mathcal{M}$ involves a partition function of the convex lattice polytope $P$ associated to $M$. We further prove that $\mathcal{M}$ is a Toeplitz operator, and as a corollary we obtain an oscillatory integral formula for the characters $\chi_{N P}$ of the torus action on $H^{0}\left(M, L^{N}\right)$.
\end{abstract}

\section{INTRODUCTION}

Toric Kähler varieties $(M, \omega)$ are often used in geometry as simple test cases for difficult geometric problems, e.g. mirror symmetry or existence of Kähler-Einstein metrics. Their simplifying features are the existence of a holomorphic action of $\left(\mathbb{C}^{*}\right)^{m}(m=\operatorname{dim} M)$ with an open dense orbit, and of a Hamiltonian action of the underlying real torus $\mathbf{T}^{m}$ with respect to the Kähler form. The associated moment map $\mu: M \rightarrow P \subset \mathbb{R}^{m}$ expresses $M$ as a stratified torus fibration over the convex lattice polytope $P$. In short, toric varieties are the completely integrable systems of complex analysis and many interesting quantities are explicitly solvable on them, often in terms of the combinatorics of $P$.

The purpose of this article is to consider toric varieties as a model setting for harmonic analysis on Kähler manifolds. Motivated by complex analysis, we consider a Hermitian holomorphic line bundle $L \rightarrow M$ with $c_{1}(L)=\omega$. Harmonic analysis in this paper refers to the Hilbert space completion of the coordinate ring,

$$
\mathcal{H}:=\bigoplus_{N=0}^{\infty} H^{0}\left(M, L^{N}\right),
$$

where $H^{0}\left(M, L^{N}\right)$ denotes the space of holomorphic sections of the $N$-th tensor power of $L$. The torus action gives rise to a natural Fourier analysis on $\mathcal{H}$. In fact, it extends to all $\mathcal{L}^{2}$ sections.

As in the classical settings of Fourier analysis, square-integrable functions on $\mathbb{R}^{n}$ and on the real $n$-torus $\mathbf{T}^{n}=\mathbb{R}^{n} / \mathbb{Z}^{n}$, the space $\mathcal{H}$ is spanned by exponentials, or more precisely, eigenfunctions of the linearized $\left(\mathbb{C}^{*}\right)^{m}$ action. In other coordinates, they appear as 'monomials.' In the fundamental case of $M=\mathbb{C P}^{m}$, the joint eigenfunctions are the monomials

Date: February 28, 2003.

Research partially supported by NSF grants DMS-0100474 (first author) and DMS-0071358 (third author) and by JSPS (second author). 
given in an affine chart by

$$
\chi_{\alpha}: \mathbb{C}^{m} \rightarrow \mathbb{C}, \quad \chi_{\alpha}(z)=z^{\alpha} .
$$

The monomials lift (by homogenization) to homogeneous monomials on $\mathbb{C}^{m+1}$. In general, the linearized $\mathbf{T}^{m}$ action is generated by $m$ commuting operators $\hat{I}_{j}, j=1, \ldots, m$ on $M$ which preserve $H^{0}\left(M, L^{N}\right)$, and the joint spectrum of the eigenvalue problem

$$
\hat{I}_{j} \varphi_{\alpha}=\alpha_{j} \varphi_{\alpha}, \quad \alpha \in \mathbb{R}^{m}, \quad \bar{\partial} \varphi_{\alpha}=0
$$

consists of lattice points $\alpha \in N P \cap \mathbb{Z}^{m}$. All of $\mathcal{L}^{2}\left(M, L^{N}\right)$ is spanned by (not necessarily holomorphic) monomials, but we will only be studying the holomorphic ones in detail.

Toric varieties are thus models of quantum completely integrable systems. They are of a very special type because integrable systems usually generate an $\mathbb{R}^{m}$ action rather than a $\mathbf{T}^{m}$ action. On such varieties, one can obtain analytical results with more precision than is possible in almost any other case. In this paper, we will illustrate this theme with regard to the Szegö kernels $\Pi_{N}=\Pi_{N}^{M}: \mathcal{L}^{2}\left(M, L^{N}\right) \rightarrow H^{0}\left(M, L^{N}\right)$.

Our first result is an exact formula for the Szegö kernel of a smooth, projective, toric Kähler variety. We shall define our toric varieties through monomial embeddings as follows (see [GKZ, Chapter 5]): Let $P$ be a convex integral polytope in $\mathbb{R}^{m}$ and denote the lattice points in $P$ by $P \cap \mathbb{Z}^{m}=\{\alpha(1), \alpha(2), \ldots \alpha(\# P)\}$. For simplicity, we shall assume throughout this paper that $P$ is contained in the positive quadrant $[0,+\infty)^{m}$. For any vector $c=$ $\left(c_{1}, \ldots, c_{\# P}\right) \in\left(\mathbb{C}^{*}\right)^{\# P}$, we define the map

$$
\Phi_{P}^{c}=\left[c_{\alpha(1)} \chi_{\alpha(1)}, \ldots, c_{\alpha(\# P)} \chi_{\alpha(\# P)}\right]:\left(\mathbb{C}^{*}\right)^{m} \rightarrow \mathbb{C P}^{\# P-1} ;
$$

i.e.,

$$
\Phi_{P}^{c}(z)=\left[c_{\alpha(1)} z^{\alpha(1)}, \ldots, c_{\alpha(\# P)} z^{\alpha(\# P)}\right] .
$$

The closure of the image is the toric variety $M_{P}^{c} \subset \mathbb{C P}^{\# P-1}$. If $P$ is Delzant, then $\Phi_{P}^{c}$ is an embedding, and we identify $\mathbb{C}^{* m}$ with its image (the 'open orbit') in $M_{P}^{c}$. To simplify the notation when $c$ and $P$ are fixed, we often write the resulting embedding as

$$
\Phi: M \hookrightarrow \mathbb{C P}^{d}, \quad d=\# P-1 .
$$

and refer to it as a monomial embedding. We further define the line bundle

$$
L=L_{P}^{c}:=\Phi^{*} \mathcal{O}(1) .
$$

Our analysis is based on identifying $\mathcal{H}$ with the Hardy space of CR functions,

$$
\mathcal{H}^{2}(X):=\left\{F \in \mathcal{L}^{2}(X): \bar{\partial}_{b} F=0\right\},
$$

where $X$ is the unit circle bundle in the dual line bundle $L^{-1}$. Its $N$-th Fourier component

$$
\mathcal{H}_{N}^{2}(X)=\left\{F \in \mathcal{H}^{2}(X): F\left(e^{i \theta} x\right)=e^{i N \theta} F(x)\right\},
$$

can be identified with $H^{0}\left(M, L^{N}\right)$ via the equivariant lifting of holomorphic sections to $X$ (see \$31). The monomial embedding $\Phi$ likewise lifts to an embedding of the $S^{1}$ bundle $X$ :

$$
\iota: X \hookrightarrow S^{2 d+1} .
$$

(See 2.2 .

Our explicit formula for the Szegö kernels $\Pi_{N}$ of the line bundles $L^{N} \rightarrow M$, i.e. the orthogonal projections onto the spaces $H^{0}\left(M, L^{N}\right)$, involves two ingredients. The first is 
the pullback of the projective space Szegö kernels of powers $\mathcal{O}(N) \rightarrow \mathbb{C P}^{\#-1}$ under the monomial embedding:

$$
\iota^{*} \Pi_{N}^{\mathbb{C P}^{d}}(x, y)=\frac{(N+m) !}{N !}\langle\iota(x), \overline{\iota(y)}\rangle^{N}, \quad x, y \in X
$$

As we will see, these simple kernels are the key objects in all of our results.

Our first result on the relation of $\Pi=\sum_{N=0}^{\infty} \Pi_{N}^{M}$ to $\Pi_{\iota}=\iota^{*} \Pi^{\mathbb{C P}^{d}}$ is valid on all integral Kähler manifolds. In Lemma 4.2, we prove that there always exists a pseudo-differential operator $A$ of order $m$ such that $\Pi \sim \Pi A \Pi \Pi_{\iota}=\Pi A \Pi_{\iota}$ modulo smoothing operators $\Pi R \Pi$. Note that this relation is equivalent to $\Pi \sim A \Pi_{\iota}$ since we can left multiply the latter by $\Pi$. Thus, only the Toeplitz part of $A$, i.e. its restriction to the Hardy space, is important in this relation. This relation might be contrasted with the comparison inequality of Li-Tian [LT], which says that the heat kernel of a Kähler manifold is less than the pull back of the projective space heat kernel under an isometric holomorphic embedding. When $t \rightarrow \infty$ this implies an inequality between $\Pi$ and $\Pi_{\iota}$.

The two kernels are more closely related on a smooth toric variety because the sections of $L_{P}^{N}$ are spanned by products of the monomials $\chi_{\alpha}$ for $\alpha \in P \cap \mathbb{Z}^{m}$. However, $\Pi_{N}$ is not equal to $\iota^{*} \Pi_{N}^{\mathbb{C P}^{d}}$ and they even have different orders as complex Fourier integral operators. But our next result shows that one may adjust one into the other using Fourier multipliers (or convolution operators) on a toric variety. The definition is analogous to the Euclidean one, namely an operator which commutes with the group action, or equivalently has the exponential functions as its eigenfunctions.

Our main results are summarized in the following theorem:

THEOREM 1.1. Let $\left(M_{P}, \omega_{P}\right)$ be a Kähler toric variety. Then there exists a Fourier multiplier $\mathcal{M}$ such that

$$
\Pi_{N}^{M_{P}}(x, y)=\mathcal{M} \cdot\langle\iota(x), \overline{\iota(y)}\rangle^{N},
$$

where $\iota: X_{P} \rightarrow S^{2 d+1}$ is the lift of the monomial embedding $\Phi$ for which $\Phi^{*} \omega_{F S}=\omega_{P}$. Moreover, $\Pi^{M_{P}} \mathcal{M} \Pi^{M_{P}}$ is a Toeplitz operator.

The Fourier multiplier is defined by the condition that its eigenvalue on a joint eigenfunction $\varphi_{\alpha} \in H^{0}\left(M_{P}, L_{P}^{N}\right)$ with $\alpha \in N P$ is given by

$$
\mathcal{P}_{N}^{-1}(\alpha) \mathcal{Q}_{N}^{-1}(\alpha)
$$

where:

(i) $\mathcal{P}_{N}$ is the lattice path 'partition function'

$$
\mathcal{P}_{N}(\alpha)=\#\left\{\left(\beta_{1}, \ldots, \beta_{N}\right): \beta_{j} \in P, \beta_{1}+\cdots+\beta_{N}=\alpha\right\} .
$$

(ii) $\mathcal{Q}_{N}(\alpha)$ is the monomial norming function:

$$
\mathcal{Q}_{N}(\alpha):=\int_{M_{P}}\left\|\chi_{\alpha}^{P}(z)\right\|_{h_{P}^{N}}^{2} d \operatorname{Vol}_{M_{P}}(z) .
$$

(Here, $\chi_{\alpha}^{P}$ is the section of $L_{P}^{N}$ corresponding to $\chi_{\alpha}$; see $\$ 2.1$.)

The statement that $\Pi \mathcal{M} \Pi$ is a Toeplitz operator means that there is a smooth symbol $\sigma$ in the usual semiclassical sense so that $\Pi \mathcal{M} \Pi=\Pi \sigma \Pi$, modulo smoothing operators. Thus, the multiplier has simple asymptotic properties, e.g. it is polyhomogeneous along rays of lattice points. The lattice path partition function seems to be of some interest in its own right. 
In a subsequent paper, we give asymptotic formulae for lattice path counting functions and applications to multiplicities of group representations [STZ2].

As a consequence of our proof of Theorem 1.1. we obtain the following integral formula for the equivariant characters $\chi_{N P}$ on $\left(\mathbb{C}^{*}\right)^{m}$ given by

$$
\chi_{N P}\left(e^{i \varphi}\right)=\sum_{\alpha \in N P} e^{i\langle\varphi, \alpha\rangle}, \quad \varphi=\left(\varphi_{1}, \ldots, \varphi_{m}\right) .
$$

THEOREM 1.2. The characters $\left\{\chi_{N P}\right\}$ are given by a complex oscillatory integral of the form

$$
\chi_{N P}\left(e^{i \varphi}\right) \sim \int_{M_{P}} e^{N \Psi(x, \varphi)} A_{N}(x) d V(x),
$$

where $\Psi$ is a non-degenerate complex phase function of positive type and $A_{N}$ is a polyhomogeneous function of $N$.

Here, ' $\sim$ ' means modulo a smoothing operator. A more precise formula is given as Proposition 5.3. We note that another oscillatory integral formula for the polytope character was given in [SZ2, Prop. 2.1] using completely different methods. In the formula in [SZ2, the amplitude is a polynomial in $N$, but depends on $\theta$ as well as $x$. Proposition 2.1 in SZ2] was used to obtain asymptotic formulas for the distribution of zeros of random polynomials with expanding Newton polytope $N P$. In fact, Proposition 5.3 provides an alternate approach to the results on zero distributions in SZ2 (for the case of Delzant polytopes) as well as to the results in our forthcoming paper [STZ1] on the distribution of values of eigenfunctions on toric varieties.

Acknowledgments: We would like to thank Amit Khetan for helpful comments concerning projective normality for toric varieties. This paper was written during a stay of the second author at Johns Hopkins University. He would like to express his special thanks to the faculty in the Department of Mathematics of Johns Hopkins University.

\section{BACKGROUND ON TORIC VARIETIES AND MOMENT POLYTOPES}

Recall that a toric variety is a complex algebraic variety $M$ containing the complex torus

$$
\mathbb{C}^{* m}:=(\mathbb{C} \backslash\{0\}) \times \cdots \times(\mathbb{C} \backslash\{0\})
$$

as a Zariski-dense open set such that the group action of $\mathbb{C}^{* m}$ on itself extends to a $\mathbb{C}^{* m}$ action on $M$. In the smooth case, $M$ can be given the structure of a symplectic manifold such that the restriction of the action to the underlying real torus

$$
\mathbf{T}^{m}=\left\{\left(\zeta_{1}, \ldots \zeta_{m}\right) \in \mathbb{C}^{* m}:\left|\zeta_{j}\right|=1,1 \leq j \leq m\right\}
$$

is a Hamiltonian action (see $(2.3)$. As mentioned in the introduction, we define projective toric varieties by the monomial embeddings (4), which depend on a convex lattice polytope $P$ and a choice of weights $c_{\alpha}$. In the smooth case, $P$ satisfies Delzant's condition (see Gu1] or [SZ2] for the definition). 
2.1. The bundles $L_{P}^{c}$ and their holomorphic sections. Recall that $H^{0}\left(\mathbb{C P}^{\# P-1}, \mathcal{O}(1)\right)$ has as a basis the linear coordinate functions $\lambda_{j}: \mathbb{C}^{\# P} \rightarrow \mathbb{C}, 1 \leq j \leq \# P$. The Fubini-study metric $h_{\mathrm{FS}}$ on $\mathcal{O}(1)$ is given by

$$
\left|\lambda_{j}\right|_{\mathrm{FS}}([\zeta])=\frac{\left|\zeta_{j}\right|}{\|\zeta\|} \quad\left(\zeta \in \mathbb{C}^{\# P}\right)
$$

which has curvature form $\omega_{\mathrm{FS}}=\frac{i}{2 \pi} \partial \bar{\partial} \log \|\zeta\|^{2}$. We endow $L_{P}^{c}$ with the Hermitian metric $h_{P}^{c}:=\Phi_{P}^{c *} h_{\mathrm{FS}}$ of curvature $\omega_{P}^{c}$ given on $\mathbb{C}^{* m}$ by

$$
\omega_{P}^{c}=\Phi_{P}^{c *} \omega_{\mathrm{FS}}=\frac{\sqrt{-1}}{2 \pi} \partial \bar{\partial} \log \sum_{\alpha \in P}\left|c_{\alpha}\right|^{2}\left|z^{\alpha}\right|^{2} .
$$

Each monomial $\chi_{\alpha}$ with $\alpha \in P$ corresponds to a section of $H^{0}\left(M_{P}^{c}, L_{P}^{c}\right)$ and vice versa. To explicitly define this correspondence, we make the identifications (recalling (6) $)$ :

$$
\chi_{\alpha(j)}^{P} \equiv c_{\alpha(j)}^{-1} \Phi_{P}^{c *} \zeta_{j} \in H^{0}\left(M_{P}^{c}, L_{P}^{c}\right), \quad 1 \leq j \leq \# P .
$$

So far, we have not specified the constants $c_{\alpha}$. For studying our phenomena, the choice of constants defining the toric variety $M_{P}$ is not important. (We use $c_{\alpha}=1$ in \$5, but except for Lemma 5.1, any choice of the $c_{\alpha}$ will work.)

More generally, a basis for the space $H^{0}\left(M_{P}, L_{P}^{N}\right)$ of global sections of $L_{P}^{N}$ is given by the monomials $\chi_{\gamma}^{P}$, where $\gamma$ runs over the lattice points of $N P$. See, for example, [Fu, $\left.\S 3.4\right]$. (In $\mathrm{Fu}$, the toric variety given by $P$ is defined using the normal fan of $P$. However by a theorem of Demazure [Fu, p. 71], the associated line bundle in this construction is very ample since $M_{P}$ is smooth, and hence the fan and monomial embedding constructions are algebraically equivalent.)

We note that since $P$ is Delzant, each $\gamma \in N P \cap \mathbb{Z}^{m}$ can be decomposed as

$$
\chi_{\gamma}^{P}=\chi_{\beta_{1}}^{P} \otimes \cdots \otimes \chi_{\beta_{N}}^{P},
$$

where $\beta_{1}, \ldots, \beta_{N} \in P \cap \mathbb{Z}^{m}$ such that $\gamma=\beta_{1}+\cdots+\beta_{N}$. Such a partition of $\gamma$ exists since $M_{P}$ is smooth and hence projectively normal [Fu, pp. 72-73]; i.e., the cone $C_{P} \subset \mathbb{C}^{\# P}$ over $M_{P}$ is normal. Indeed, by normality of $C_{P}$, each section of $H^{0}\left(M_{P}, L_{P}^{N}\right)$ corresponds to a homogeneous polynomial of order $N$ on $\mathbb{C}^{\# P}$; i.e., $H^{0}\left(M_{P}, L_{P}\right)$ generates the coordinate ring $\bigoplus_{N=1}^{\infty} H^{0}\left(M_{P}, L_{P}^{N}\right)$, and (13) follows.

Remark: For a general integral polytope $P$, (13) may not hold. A well-known example is where $P$ is the simplex in $\mathbb{R}^{3}$ with vertices $(0,0,0),(0,1,1),(1,0,1),(1,1,0)$. Then $P \cap \mathbb{Z}^{3}$ consists only of the vertices, and for all $N \geq 2$, the lattice point $\gamma=(1,1,1)$ lies in $N P$ but cannot be decomposed as in (13).

2.2. Lifting to the associated $S^{1}$ bundle. The geometry of line bundles can be rephrased in terms of the associated principal $S^{1}$ bundle

$$
X_{P}=\left\{v \in L_{P}^{-1}:\|v\|_{h_{P}^{-1}}=1\right\},
$$

where $L_{P}^{-1} \rightarrow M_{P}$ denote the dual line bundle to $L_{P}$ with dual metric $h_{P}^{-1}$.

The action of the real torus $\mathbf{T}^{m}$ lifts from $M_{P}^{c}$ to $X_{P}^{c}$ and combines with the $S^{1}$ action to define a $\mathbf{T}^{m+1}$ action on $X_{P}^{c}$. Recall that under the monomial embedding

$$
\Phi_{P}^{c}: \mathbb{C}^{* m} \hookrightarrow M_{P}^{c} \hookrightarrow \mathbb{C P}^{\# P-1}, \quad z \mapsto\left[c_{\alpha(1)} z^{\alpha(1)}, \ldots, c_{\alpha(\# P)} z^{\alpha(\# P)}\right],
$$


the $\mathbf{T}^{m}$ action on $M_{P}^{c} \subset \mathbb{C P} \# P-1$ is given by

$$
e^{i \varphi} \cdot\left[\zeta_{1}, \ldots, \zeta_{\# P}\right]=\left[e^{i\langle\alpha(1), \varphi\rangle} \zeta_{1}, \ldots, e^{i\langle\alpha(\# P), \varphi\rangle} \zeta_{\# P}\right], \quad e^{i \varphi}=\left(e^{i \varphi_{1}}, \ldots, e^{i \varphi_{m}}\right) .
$$

The action (14) lifts to an action on $L_{P}^{-1}$ :

$$
e^{i \varphi} \cdot \zeta=\left(e^{i\langle\alpha(1), \varphi\rangle} \zeta_{1}, \ldots, e^{i\langle\alpha(\# P), \varphi\rangle} \zeta_{\# P}\right)
$$

Since the circle bundle $X_{P}^{c} \subset S^{2 \# P-1}$ is invariant under this action, (15) also gives a lift of the action (14) to $X_{P}^{c}$.

We also have the standard circle action on $X_{P}^{c}$ :

$$
e^{i \theta} \cdot \zeta=e^{i \theta} \zeta
$$

which commutes with the $\mathbf{T}^{m}$-action (15). Combining (15) and (16), we then obtain a $\mathbf{T}^{m+1}$-action on $X_{P}^{c}$ :

$$
\left(e^{i \theta}, e^{i \varphi_{1}}, \ldots, e^{i \varphi_{m}}\right) \bullet \zeta=e^{i \theta}\left(e^{i \varphi} \cdot \zeta\right) .
$$

We identify sections $s_{N}$ of $L^{N}$ with equivariant functions $\hat{s}_{N}$ on $X$ by the rule

$$
\hat{s}_{N}(\lambda)=\left(\lambda^{\otimes N}, s_{N}(z)\right), \quad \lambda \in X_{z},
$$

where $\lambda^{\otimes N}=\lambda \otimes \cdots \otimes \lambda$. Clearly, $\hat{s}_{N}\left(e^{i \theta} \cdot x\right)=e^{i N \theta} \hat{s}_{N}(x)$ if $s \in H^{0}\left(M_{P}^{c}, L_{P}^{N}\right)$.

It should be noted that, for each $s \in H^{0}\left(M_{P}, L_{P}^{N}\right)$, we have

$$
\left|\widehat{s}_{N}(x)\right|=\|s(z)\|_{h_{P}^{N}} .
$$

We now introduce notation for the lifts of monomials: for $\alpha \in P$, we lift $\chi_{\alpha}^{P} \in H^{0}\left(M_{P}^{c}, L_{P}^{c}\right)$ to an equivariant function $\widehat{\chi}_{\alpha}^{P}$ on the circle bundle $X_{P}^{c} \rightarrow M_{P}^{c}$, and we write

$$
\widehat{m}_{\alpha(j)}^{P}:=c_{\alpha(j)} \widehat{\chi}_{\alpha(j)}^{P}=\zeta_{j} \circ \iota_{P}
$$

where $\iota_{P}: X_{P}^{c} \rightarrow S^{2 d+1}$ is the lift of the embedding $M_{P}^{c} \hookrightarrow \mathbb{C P}^{d}(d=\# P-1)$. (Of course, $\widehat{m}_{\alpha}^{P}$ depends on $c$, which we omit to simplify notation.) We also consider the monomials

$$
m_{\alpha}^{P}:=c_{\alpha} \chi_{\alpha}^{P}
$$

so that $\widehat{m}_{\alpha}^{P}$ is the equivariant lift of $m_{\alpha}$ to $X_{P}^{c}$. In terms of local coordinates $(z, \theta)$ on $\pi^{-1}\left(\mathbb{C}^{* m}\right) \subset X_{P}^{c}$, we have

$$
\widehat{m}_{\alpha}^{P}(z, \theta)=\frac{e^{i \theta} c_{\alpha} z^{\alpha}}{\left(\sum_{\beta \in P}\left|c_{\beta}\right|^{2}\left|z^{\beta}\right|^{2}\right)^{1 / 2}} .
$$

2.3. Moment maps and torus actions. The group $\mathbb{C}^{* m}$ acts on $M_{P}^{c}$ and the subgroup $\mathbf{T}^{m}$ acts in a Hamiltonian fashion. Let us recall the formula for its moment map $\mu_{P}^{c}: M_{P}^{c} \rightarrow \mathbb{R}^{m}$, restricted to the open orbit $\mathbb{C}^{* m}$. This moment map is the composition

$$
\mu_{P}^{c}: M_{P}^{c} \subset \mathbb{C P}^{\# P-1} \stackrel{\mu_{0}}{\rightarrow} \mathbb{R}^{\# P} \stackrel{A}{\rightarrow} \mathbb{R}^{m},
$$

where

$$
\mu_{0}\left(\left[z_{1}, \ldots, z_{\# P}\right]\right)=\frac{1}{\|z\|^{2}}\left(\left|z_{1}\right|^{2}, \ldots,\left|z_{\# P}\right|^{2}\right)
$$

and $A$ is the linear projection is given by the column vectors $\left(\alpha^{1}, \ldots, \alpha^{\# P}\right)$. Hence we have

$$
\mu_{P}^{c}=\frac{1}{\sum_{\alpha \in P}\left|c_{\alpha}\right|^{2}\left|\chi_{\alpha}\right|^{2}} \sum_{\alpha \in P}\left|c_{\alpha}\right|^{2}\left|\chi_{\alpha}\right|^{2} \alpha .
$$


For any $c$, the image of $M_{P}^{c}$ under $\mu_{P}^{c}$ equals $P$.

Noting that

$$
\sum_{\alpha \in P}\left|\widehat{m}_{\alpha}^{P}\right|^{2}=\sum_{j=1}^{\# P}\left|\zeta_{j} \circ \iota_{P}\right|^{2} \equiv 1
$$

we obtain the formula:

$$
\mu_{P}^{c}(z)=\sum_{\alpha \in P}\left|\widehat{m}_{\alpha}^{P}(z)\right|^{2} \alpha
$$

(We write $\left|\widehat{m}_{\alpha}^{P}(z)\right|=\left|\widehat{m}_{\alpha}^{P}(z, \theta)\right|$, since the absolute value is independent of $\theta$.)

2.3.1. Projective space. We illustrate with the case of $\mathbb{C P}^{m}$ and introduce notation that will be used throughout the paper. As a toric variety, $\mathbb{C P}^{m}=M_{\Sigma}$, where $\Sigma$ is the standard simplex in $\mathbb{R}^{m}$ with vertices at the points

$$
(0, \ldots, 0),(1,0, \ldots, 0),(0,1, \ldots, 0), \ldots,(0, \ldots, 0,1),
$$

and $L_{\Sigma}$ is the hyperplane section bundle $\mathcal{O}(1) \rightarrow \mathbb{C P}^{m}$. In this case, $X=S^{2 m+1}$ and the lifts of sections in $H^{0}\left(\mathbb{C P}^{m}, \mathcal{O}(p)\right)$ to $X$ consist of homogeneous polynomials

$$
F\left(\zeta_{0}, \ldots, \zeta_{m}\right)=\sum_{|\lambda|=p} C_{\lambda} \zeta^{\lambda} \quad\left(\zeta^{\lambda}=\zeta_{0}^{\lambda_{0}} \cdots \zeta_{m}^{\lambda_{m}}\right)
$$

in $m+1$ variables. We give $\mathbb{C P}^{m}$ the Fubini-Study Kähler form given in homogeneous coordinates $\left(\zeta_{0}, \ldots, \zeta_{m}\right)$ by $\omega_{\mathrm{FS}}=\frac{i}{2 \pi} \partial \bar{\partial} \log \|\zeta\|^{2}$, and we give $\mathcal{O}(p)$ the Fubini-Study metric:

$$
|F(\zeta)|_{\mathrm{FS}}=|F(\zeta)| /\|\zeta\|^{p}, \quad \text { for } F \in H^{0}\left(\mathbb{C P}^{m}, \mathcal{O}(p)\right) \text {. }
$$

Identifying $F$ with the polynomial $f(z)=F\left(1, z_{1}, \ldots, z_{m}\right)$, the Fubini-Study norm can be written

$$
|f(z)|_{\mathrm{FS}}=|f(z)| /\left(1+\|z\|^{2}\right)^{p / 2} \quad\left(z \in \mathbb{C}^{m}\right) .
$$

We equip the space $H^{0}\left(\mathbb{C} \mathbb{P}^{m}, \mathcal{O}(p)\right)$ of all homogeneous polynomials of degree $p$ with the Hermitian inner product:

$$
\langle f, \bar{g}\rangle=\int_{\mathbb{C P}^{m}}\langle F, \bar{G}\rangle_{\mathrm{FS}} d \operatorname{Vol}_{\mathbb{C P}^{m}}=\frac{1}{m !} \int_{\mathbb{C}^{m}} \frac{\langle f(z), \overline{g(z)}\rangle}{\left(1+\|z\|^{2}\right)^{p}} \omega_{\mathrm{FS}}^{m}(z), \quad f, g \in H^{0}\left(\mathbb{C P}^{m}, \mathcal{O}(p)\right) .
$$

(We use here the Riemannian volume $d \mathrm{Vol}_{\mathbb{C P}^{m}}=\frac{1}{m !} \omega_{\mathrm{FS}}^{m}$; note that the total volume of $\mathbb{C P}^{m}$ is $\frac{1}{m !}$, using our conventions.)

Under the $\mathbf{T}^{m}$ action, we have the weight space decomposition

$$
H^{0}\left(\mathbb{C P}^{m}, \mathcal{O}(p)\right)=\bigoplus_{|\alpha| \leq p} \mathbb{C} \chi_{\alpha}
$$

where we recall that $\chi_{\alpha}(z)=z_{1}^{\alpha_{1}} \cdots z_{m}^{\alpha_{m}}$. The monomials $\left\{\chi_{\alpha}\right\}$ are orthogonal but not normalized. Any choice of norming constants $\left\{r_{\alpha} \in \mathbb{C}^{*}\right\}$ will give a monomial basis $\left\{r_{\alpha} \chi_{\alpha}\right\}$ for $H^{0}\left(\mathbb{C P} \mathbb{P}^{m}, \mathcal{O}(p)\right)$. We shall choose $r_{\alpha}=\left\|\chi_{\alpha}\right\|_{\mathbb{C P} m}^{-1}$, where

$$
\left\|\chi_{\alpha}\right\|_{\mathbb{C P}^{m}}=\sqrt{\left\langle\chi_{\alpha}, \chi_{\alpha}\right\rangle}=\left[\frac{p !}{(p+m) !\left(\begin{array}{c}
p \\
\alpha
\end{array}\right)}\right]^{\frac{1}{2}}, \quad\left(\begin{array}{l}
p \\
\alpha
\end{array}\right):=\frac{p !}{(p-|\alpha|) ! \alpha_{1} ! \cdots \alpha_{m} !},
$$


is the Fubini-Study $\mathcal{L}^{2}$ norm of $\chi_{\alpha}$ given by (25). (See [SZ1, $\left.\S 4.2\right]$; the extra factor $m$ ! in SZ1 is due to the use of $\omega^{m}$ instead of $\frac{\omega^{m}}{m !}$ for the volume form.) This choice provides an orthonormal basis for $H^{0}\left(\mathbb{C P}^{m}, \mathcal{O}(p)\right)$ given by the monomials

$$
\frac{1}{\left\|\chi_{\alpha}\right\|_{\mathbb{C P}^{m}}} \chi_{\alpha}=\sqrt{\frac{(p+m) !}{p !}\left(\begin{array}{l}
p \\
\alpha
\end{array}\right)} \chi_{\alpha}, \quad|\alpha| \leq p .
$$

In this case, we shall use the special choice of the coefficients of the monomial embedding

$$
c_{\alpha}^{*}:=\left(\begin{array}{c}
p \\
\alpha
\end{array}\right)^{\frac{1}{2}}=\left(\frac{p !}{(p+m) !}\right)^{\frac{1}{2}}\left\|\chi_{\alpha}\right\|_{\mathbb{C P} m}^{-1}
$$

so that

$$
\mu_{p \Sigma}(z):=\mu_{p \Sigma}^{c^{*}}(z)=\frac{1}{\sum_{|\alpha| \leq p}\left(\begin{array}{c}
p \\
\alpha
\end{array}\right)\left|z^{\alpha}\right|^{2}} \sum_{|\alpha| \leq p}\left(\begin{array}{c}
p \\
\alpha
\end{array}\right)\left|z^{\alpha}\right|^{2} \alpha=\frac{p}{1+\sum\left|z_{j}\right|^{2}}\left(\left|z_{1}\right|^{2}, \ldots,\left|z_{m}\right|^{2}\right),
$$

where the last equality follows by differentiating the identity $\left(1+\sum x_{j}\right)^{p}=\sum_{|\alpha| \leq p}\left(\begin{array}{l}p \\ \alpha\end{array}\right) x^{\alpha}$. Note that this choice gives us the scaling formula

$$
\mu_{p \Sigma}=p \mu_{\Sigma}
$$

We can identify $L^{-1}=\mathcal{O}_{\mathbb{C P} m}(-1)$ with $\mathbb{C}^{m+1}$ with the origin blown up, and the circle bundle $X \subset L^{-1}$ is identified with the unit sphere $S^{2 m+1} \subset \mathbb{C}^{m+1}$. Then the equivariant lift $\widehat{\chi}_{\alpha}: S^{2 m+1} \rightarrow \mathbb{C}$ of $\chi_{\alpha} \in H^{0}\left(\mathbb{C P}^{m}, \mathcal{O}(p)\right)$ is given by the homogenization:

$$
\widehat{\chi}_{\alpha}(x)=x^{\widehat{\alpha}}, \quad \widehat{\alpha}=\left(\alpha_{1}, \ldots, \alpha_{m}, p-|\alpha|\right) .
$$

Furthermore,

$$
\left|\widehat{m}_{\alpha}^{p \Sigma}(z)\right|=\left[\frac{p !}{(m+p) !}\right]^{\frac{1}{2}} \frac{\left|\widehat{\chi}_{\alpha}(z)\right|}{\left\|\chi_{\alpha}\right\|_{\mathbb{C P} m}}
$$

where, by abuse of notation, we regard $\left|\widehat{\chi}_{\alpha}\right|$ and $\left|\widehat{m}_{\alpha}^{p \Sigma}\right|$ as functions on $\mathbb{C}^{* m}$, since they are invariant under the circle action. Strictly speaking, the $S^{1}$-bundle $X_{p \Sigma}$ in this case is given by $X_{p \Sigma} \equiv S^{2 m+1} / \mathbb{Z}_{p}$. Then we have the more precise formula

$$
\widehat{m}_{\alpha}^{p \Sigma}\left(x^{\prime}\right)=\left[\frac{p !}{(m+p) !}\right]^{\frac{1}{2}} \frac{\widehat{\chi}_{\alpha}(x)}{\left\|\chi_{\alpha}\right\|_{\mathbb{C P}^{m}}},
$$

where $x^{\prime} \in M_{p \Sigma}$ is the equivalence class of $x \in S^{2 m+1}$, and therefore we have

$$
\sum_{|\alpha| \leq p} \widehat{m}_{\alpha}^{p \Sigma}\left(x^{\prime}\right) \overline{\widehat{m}_{\alpha}^{p \Sigma}\left(y^{\prime}\right)}=\langle x, \bar{y}\rangle^{p} .
$$

\section{Fourier ANALYSIS}

As mentioned in the introduction, we identify $\mathcal{H}$ with the Hardy space $\mathcal{H}^{2}\left(X_{P}^{c}\right)$. It is a Hilbert space with the inner product

$$
\langle f, \bar{g}\rangle=\int_{X} f \bar{g} d \mathrm{Vol}_{X}, \quad d \mathrm{Vol}_{X}=\frac{1}{m !} \alpha \wedge(d \alpha)^{m},
$$


where $\alpha$ is a contact 1 -form defined by the Hermitian connection on $L_{P}^{-1}$ such that $d \alpha=\pi^{*} \omega_{P}$. Under the identification of sections with equivariant functions, the inner product is the same as

$$
\left\langle s_{1}, \bar{s}_{2}\right\rangle_{N}=\int_{M}\left\langle s_{1}(z), \overline{s_{2}(z)}\right\rangle_{h_{P}^{N}} d \operatorname{Vol}_{M}(z), \quad s_{1}, s_{2} \in H^{0}\left(M_{P}, L_{P}^{N}\right) .
$$

Under the $S^{1}$ action, the Hardy space has the orthogonal decomposition

$$
\mathcal{H}^{2}\left(X_{P}^{c}\right)=\bigoplus_{N=0}^{\infty} \mathcal{H}_{N}^{2}\left(X_{P}^{c}\right)
$$

We recall that the (equivariant) 'Szegö projectors' $\Pi_{N}$ are the orthogonal projection onto $H^{0}\left(M_{P}^{c}, L_{P}^{c N}\right)$. If $\left\{S_{j}^{N}\right\}$ denotes an orthonormal basis of this space, and $\hat{S}_{j}{ }^{N}$ denote their lifts to $X$, then the projector $\Pi_{N}$ is given by the kernel

$$
\Pi_{N}(x, y)=\sum_{j=1}^{k_{N}} \hat{S}_{j}^{N}(z) \overline{\hat{S}_{j}^{N}(y)}: \mathcal{L}^{2}\left(X_{P}^{c}\right) \rightarrow \mathcal{H}_{N}^{2}\left(X_{P}^{c}\right) .
$$

The full Szegö kernel is the equivariant direct sum

$$
\Pi=\sum_{N=1}^{\infty} \Pi_{N}: \mathcal{L}^{2}\left(X_{P}^{c}\right) \rightarrow \mathcal{H}^{2}\left(X_{P}^{c}\right) .
$$

For each $N \in \mathbb{N}$, we define the 'homogenization' $\widehat{N P} \subset \mathbb{Z}^{m+1}$ of the lattice point in the polytope $N P$ to be the set of all lattice point $\widehat{\alpha}^{N}$ of the form

$$
\widehat{\alpha}^{N}=\widehat{\alpha}:=\left(\alpha_{1}, \ldots, \alpha_{m}, N p-|\alpha|\right), \quad \alpha=\left(\alpha_{1}, \ldots, \alpha_{m}\right) \in N P \cap \mathbb{Z}^{m},
$$

where, as before, we set $p=\max _{\beta \in P \cap \mathbb{Z}^{m}}|\beta|$. We also define the cone $\Lambda_{P}=\bigcup_{N=1}^{\infty} \widehat{N P}$.

In this section, we consider the lifted monomials by $\widehat{\chi}_{\widehat{\alpha}}(x)=\widehat{\chi}_{\alpha}(x)$, for $\widehat{\alpha} \in \Lambda_{P}$. We may combine the problems as $N$ varies into a homogeneous eigenvalue problem on $X$ : After homogenization, i.e. lifting to $X$, we obtain the joint scalar eigenvalue problem

$$
\hat{I}_{j} \widehat{\chi}_{\widehat{\alpha}}=\widehat{\alpha}_{j} \widehat{\chi}_{\widehat{\alpha}}, \quad \widehat{\alpha} \in \mathbb{R}^{m+1}, \quad \bar{\partial}_{b} \widehat{\chi}_{\widehat{\alpha}}=0, \quad j=1, \ldots, m+1 .
$$

The lattice points $\widehat{\alpha}$ lie in the cone $\Lambda_{P} \subset \mathbb{R}^{m+1}$. It is well known that rays $\mathbb{N} \widehat{\alpha}$ in this cone define a semiclassical limit.

The torus action on $X_{P}^{c}$ can be quantized to define an action of the torus as unitary operators on $\mathcal{H}^{2}\left(X_{P}^{c}\right)$. Specifically, we let $\Xi_{1}, \ldots \Xi_{m}$ denote the differential operators on $X_{P}^{c}$ generated by the $\mathbf{T}^{m}$ action:

$$
\left(\Xi_{j} \hat{S}\right)(\zeta)=\left.\frac{1}{i} \frac{\partial}{\partial \varphi_{j}} \hat{S}\left(e^{i \varphi} \cdot \zeta\right)\right|_{\varphi=0}, \quad \hat{S} \in \mathcal{C}^{\infty}\left(X_{P}^{c}\right)
$$

Proposition 3.1. For $1 \leq j \leq m$,

(i) $\Xi_{j}: \mathcal{H}_{N}^{2}\left(X_{P}^{c}\right) \rightarrow \mathcal{H}_{N}^{2}\left(X_{P}^{c}\right)$;

(ii) The lifted monomials $\hat{\chi}_{\alpha}^{P} \in \mathcal{H}_{N}^{2}\left(X_{P}^{c}\right)$ satisfy $\Xi_{j} \hat{\chi}_{\alpha}^{P}=\alpha_{j} \hat{\chi}_{\alpha}^{P}(\alpha \in N P)$.

Proof. Item (i) follows from the fact that the $\mathbf{T}^{m}$ action is holomorphic and commutes with $\frac{\partial}{\partial \theta}$. For the case $N=1$, (ii) follows immediately from (20) and (15). For $\alpha \in N P, N>1$, we write $\alpha=\beta^{1} \cdots \beta^{N}$ with $\beta^{k} \in P$, and the conclusion then follows from the first case and the product rule. 
Furthermore, we recall that

$$
\frac{\partial}{\partial \theta}: \mathcal{H}_{N}^{2}\left(X_{P}^{c}\right) \rightarrow \mathcal{H}_{N}^{2}\left(X_{P}^{c}\right), \quad \frac{1}{i} \frac{\partial}{\partial \theta} \hat{s}_{N}=N \hat{s}_{N} \quad \text { for } \hat{s}_{N} \in \mathcal{H}_{N}^{2}\left(X_{P}^{c}\right) .
$$

Thus, we have the joint eigenvalue problem (33) with the commuting operators:

$$
\hat{I}_{j}=\Xi_{j}, \quad j=1, \ldots, m, \quad \hat{I}_{m+1}=\frac{p}{i} \frac{\partial}{\partial \theta}-\sum_{j=1}^{m} \Xi_{j} .
$$

The monomials $\widehat{\chi}_{\hat{\alpha}}$ are the joint eigenfunctions of $\left(\hat{I}_{1}, \ldots, \hat{I}_{m+1}\right)$ for the joint eigenvalues $\widehat{\alpha} \in \Lambda_{P}$.

Remark: The vector fields $\Xi_{j}$ can be constructed geometrically as follows (see [Gu1]): Let $\xi_{j}=\frac{\partial}{\partial \varphi_{j}}(1 \leq j \leq m)$ denote the Hamiltonian vector fields generating the $\mathbf{T}^{m}$ action on $M_{P}^{c}$. There is a natural contact 1 -form $\alpha$ on $X_{P}^{c}$ determined by the Hermitian connection; a key property of $\alpha$ is that $d \alpha=\pi^{*} \omega$ (see [Ze]). We use $\alpha$ to define the horizontal lifts of the Hamilton vector fields $\xi_{j}$ :

$$
\pi_{*} \xi_{j}^{h}=\xi_{j}, \quad \alpha\left(\xi_{j}^{h}\right)=0 .
$$

The vector fields $\Xi_{j}$ are then given by:

$$
\Xi_{j}=\xi_{j}^{h}+2 \pi i\left\langle\mu_{P}^{c} \circ \pi, \xi_{j}^{*}\right\rangle \frac{\partial}{\partial \theta}=\xi_{j}^{h}+2 \pi i\left(\mu_{P}^{c} \circ \pi\right)_{j} \frac{\partial}{\partial \theta} .
$$

(Here, $\xi_{j}^{*} \in \mathbb{R}^{m}$ is the element of the Lie algebra of $\mathbf{T}^{m}$ which acts as $\xi_{j}$ on $M_{P}$.)

3.1. Fourier and Toeplitz analysis on toric varieties. Under the lifted torus action of $\mathbf{T}^{m}$ on $X_{P}$ generated by the $\Xi_{j}$, we can further decompose $\mathcal{L}^{2}\left(X_{P}^{c}\right)$ and $\mathcal{H}^{2}\left(X_{P}^{c}\right)$ into representations of $\mathbf{T}^{m}$. Since the action commutes with $S^{1}$, we combine the two actions into an action of $\mathbf{T}^{m+1}$ on $X_{P}$.

The $\mathbf{T}^{m+1}$ action on $\mathcal{H}^{2}$ is multiplicity free and we have

$$
\mathcal{H}^{2}\left(X_{P}^{c}\right)=\bigoplus_{\hat{\alpha} \in \Lambda_{P}} \mathbb{C} \hat{\varphi}_{\hat{\alpha}}
$$

We will also need to decompose the action on $X_{P}$. To this end, we introduce the anti-Hardy space $\overline{\mathcal{H}}^{2}\left(X_{P}^{c}\right)$ of anti-CR functions, i.e. solutions of $\partial_{b} f=0$. Of course, a Hilbert basis is given by the complex-conjugate monomials $\overline{\hat{\chi}}_{\hat{\alpha}}$.

Because the $\mathbf{T}^{m+1}$ action is generated by vector fields, products of eigenfunctions are also eigenfunctions. Hence, the orthonormal mixed monomials

$$
\hat{\chi}_{\hat{\alpha}, \hat{\beta}}(x)=\hat{\chi}_{\hat{\alpha}} \overline{\hat{\chi}}_{\hat{\beta}}
$$

are eigenfunctions of eigenvalue $\hat{\alpha}-\hat{\beta}$ for $\left\{\hat{I}_{1}, \ldots, \hat{I}_{m+1}\right\}$ defined in (36). We now claim that these mixed monomials furnish a Hilbert basis of $\mathcal{L}^{2}\left(X_{P}^{c}\right)$.

Proposition 3.2. We have: $\mathcal{L}^{2}\left(X_{P}^{c}\right)=\bigoplus_{\hat{\alpha}, \hat{\beta} \in \Lambda_{P}} \mathbb{C} \hat{\chi}_{\hat{\alpha}, \hat{\beta}}$.

Proof. It suffices to show that the closure of the algebra generated by the $\hat{\chi}_{\alpha, \beta}$ equals the space $C\left(X_{P}^{c}\right)$ of continuous functions on $X_{P}$. Since the span of the monomials is a *algebra, it suffices by Stone-Weierstrass to show that such polynomials separate points. But this 
is clear since $L_{P}^{c}$ is ample: for any $z \in M_{P}$ there exists $N, s_{N} \in H^{0}\left(M_{P}^{c}, L_{P}^{c N}\right)$ such that $\left|s_{N}(z)\right| \neq 0$. It follows that $\left|\hat{s}_{N}(x)\right| \neq 0$ for any $x$ over $z$.

It follows that the joint spectrum of $\left(\hat{I}_{1}, \ldots, \hat{I}_{m+1}\right)$ is given by

$$
\operatorname{Spec}\left(\hat{I}_{1}, \ldots, I_{m+1}\right)=\Lambda_{P}-\Lambda_{P}=\mathbb{Z}^{m+1} .
$$

However, the multiplicity of a lattice point $\hat{\gamma} \in \mathbb{Z}^{m+1}$ is infinite since any $(\hat{\alpha}, \hat{\beta})$ with $\hat{\alpha}-\hat{\beta}=\hat{\gamma}$ corresponds to an eigenvector. This reflects the fact that $\left(\Xi_{1}, \ldots, \Xi_{m}, \frac{\partial}{\partial \theta}\right)$ is not an elliptic system.

3.2. Fourier multipliers and Toeplitz Fourier multipliers. We now define the analogues of convolution operators or Fourier multipliers:

Definition: An operator $\Pi F \Pi$ on $\mathcal{H}^{2}\left(X_{P}\right)$ will be called a Fourier multiplier if it satisfies the following (equivalent) conditions:

- $F$ may be expressed as a function $F(D)$ of the commuting system of operators $D=$ $\left(\Xi_{1}, \ldots, \Xi_{m}, \frac{\partial}{\partial \theta}\right)$.

- Its eigenfunctions are the monomials $\hat{\chi}_{\hat{\alpha}, \hat{\beta}}$.

As emphasized above, our main interest is in holomorphic functions. The relevant definition is:

Definition: An operator $\Pi F \Pi$ on $\mathcal{H}^{2}\left(X_{P}\right)$ will be called a Toeplitz Fourier multiplier if its eigenfunctions are the holomorphic monomials $\hat{\chi}_{\hat{\alpha}}^{P}$.

It is obvious that $\Pi F \Pi$ is a Toeplitz Fourier multiplier if $F$ is a Fourier multiplier, but the converse is obviously not necessarily true. In fact, it is sometimes not obvious how to extend a Toeplitz Fourier multiplier to all of $\mathcal{L}^{2}(X)$ and to characterize it there.

\section{Pull Back of the Projective space Szegö Kernel}

Theorem 1.1 gives a relation between the Szegö kernel of a toric variety and the very simple kernel obtained by pulling back the projective space Szegö kernel under a holomorphic embedding. As mentioned in the introduction, the pull back kernels are the key objects in the proofs of all of our results. In this section, we study the pull back kernel on any positive line bundle over any Kähler manifold, and express it as a modification of the Szegö kernel. In the next section, we will show that on a toric variety this relation can be inverted to give a construction of the Szegö kernel.

Consider any polarized algebraic manifold $(M, L)$, and assume $L$ is very ample. Choose a basis $\left\{S_{0}, \ldots, S_{d}\right\}$ of $H^{0}(M, L)$ and let $\Phi$ denote the associated embedding into projective space. That is, we write $S_{j}=f_{j} e_{L}$ relative to a local frame $e_{L}$ and put $\Phi(z)=$ $\left[f_{0}(z), \ldots, f_{d}(z)\right]$. Recalling that $L=\Phi^{*} \mathcal{O}(1)$, we can equip $L$ with the metric $\Phi^{*} h_{\mathrm{FS}}$. We also give $M$ the metric $\omega=\Phi^{*} \omega_{\mathrm{FS}}$, and we let $\Pi_{N}$ denote the orthogonal projection onto $H^{0}\left(M, L^{N}\right)$ with respect to these metrics. Also, let $\Pi=\sum_{N=1}^{\infty} \Pi_{N}$ denote the Szegö kernel.

The embedding $\Phi$ determines the lift $\iota: X \rightarrow S^{2 d+1}$. Recalling (8), we let

$$
\Pi_{\iota}(x, y)=\Pi(\iota(x), \iota(y))=\sum \Pi_{N}^{\mathbb{C P}^{d}}(\iota(x), \iota(y))=\frac{d !}{(1-\langle\iota(x), \overline{\iota(y)}\rangle)^{d+1}}, \quad x, y \in X
$$


denote the pullback to $X$ of the Szegö kernel of $\mathcal{O}(1) \rightarrow \mathbb{C P}^{d}$. The question we study in this section is the relation of this simple kernel to the Szegö kernel $\Pi=\Pi^{M}$ with respect to $\iota^{*} \omega_{\text {FS }}$. The following proposition shows that $\Pi_{\iota}$ is a Toeplitz operator. We assume basic knowledge of such operators and their symbols (see e.g. Gu2]). We denote by $S^{k}(M, \mathbb{N}$ ) the space of semiclassical symbols $s: M \times \mathbb{N} \rightarrow \mathbb{C}$ of the form

$$
s_{N}(z) \sim N^{k} \sum_{j=0}^{\infty} s_{-j}(z) N^{-j}
$$

We let

$$
\widetilde{\Pi}_{1}^{M}(x, y):=\frac{1}{d+1} \Pi_{1}^{\mathbb{C P}^{d}}(\iota(x), \iota(y))=\langle\iota(x), \overline{\iota(y)}\rangle .
$$

For simplicity of notation, we shall write

$$
\Pi_{1}^{N}(x, y)=\left[\widetilde{\Pi}_{1}^{M}(x, y)\right]^{N} .
$$

We note that $\Pi_{1}^{N}(x, x)=1$.

Proposition 4.1. Let $(M, L)$ be a polarized algebraic manifold as above. Then there exists a semi-classical symbol $a_{N} \in S^{-m}(M, \mathbb{N})$ with principal symbol $s_{-m}=1$ so that

$$
\Pi_{1}^{N}=\Pi_{N} a_{N} \Pi_{N}+R_{N} \quad \text { where }\left\|R_{N}\right\|_{\mathrm{HS}}=O\left(N^{-k}\right) \quad \forall k .
$$

Here, $\left\|R_{N}\right\|_{\mathrm{HS}}^{2}=$ Trace $R_{N}^{*} R_{N}$.

This proposition will be used in the proof of Theorem 1.1. The main step in the proof expresses $\Pi$ as the composition of $\Pi_{\iota}$ and of a Toeplitz operator.

LEMma 4.2. Let $\Pi$ denote the Szegö projector associated to the metric $\iota^{*} \omega_{\mathrm{FS}}$. Then, there exist $A \in \Psi^{m}(X)$ with $\left[A, D_{\theta}\right]=0$ such that $\Pi \sim \Pi A \Pi \Pi_{\iota}=\Pi A \Pi_{\iota}$ modulo smoothing operators $\Pi R \Pi$.

Here, $\Psi^{m}(X)$ denotes the space of pseudodifferential operators of order $m$ on $X$. An operator of the form $\Pi A \Pi$ where $A \in \Psi^{m}(X)$ for some $m$ is a Toeplitz operator in the sense of $[\mathrm{BG}$. A smoothing Toeplitz operator is a smoothing operator of the form $\Pi R \Pi$. It follows then that $\Pi_{N}=T_{N} \Pi_{1}^{N}$ where $T_{N}$ is a Toeplitz operator.

4.0.1. The projective Szegö kernels. Since our formula for the Szegö kernel of a general toric variety involves the pullback of the projective space Szegö kernel, we recall how the latter is defined. We note that

$$
\widehat{\chi}_{\alpha}(x)=x^{\hat{\alpha}^{p}}
$$

and hence the Szegö kernel $\Pi_{p}^{\mathbb{C P}}{ }^{m}$ for the orthogonal projection is given by:

$$
\Pi_{p}^{\mathbb{C P}^{m}}(x, y)=\sum_{|\alpha| \leq p} \frac{1}{\left\|\chi_{\alpha}\right\|_{\mathbb{C P} m}} \widehat{\chi}_{\alpha}(x) \overline{\widehat{\chi}_{\alpha}(y)}=\frac{(p+m) !}{p !} \sum_{|\alpha| \leq p}\left(\begin{array}{c}
p \\
\alpha
\end{array}\right) x^{\hat{\alpha}^{p}} \bar{y}^{\hat{\beta}^{p}}=\frac{(p+m) !}{p !}\langle x, \bar{y}\rangle^{p}
$$

for $x, y \in S^{2 m+1}$. (The sum $\sum_{p=0}^{\infty} \Pi_{p}^{\mathbb{C P}^{m}}$ is the usual Szegö kernel for the sphere.)

Note that when $M_{\Sigma}=M_{p \Sigma}=\mathbb{C P}^{m}$, we have the circle bundles $X_{p \Sigma} \rightarrow \mathbb{C P}^{m}$, for $p \geq 1$. When $p=1, X_{\Sigma}=S^{2 m+1}$ while for $p>1$, it is the lens space $X_{p \Sigma}=S^{2 m+1} /\left\{e^{2 \pi i / p}\right\}$. The latter statement follows from the fact that homogeneous polynomials of degree $p$ are well defined on (and separate points of) the quotient by the cyclic group of $p$-th roots of unity. 
4.0.2. Boutet de Monvel-Sjöstrand parametrix. To prove Lemma 4.2, we need to recall some background on parametrices for $\Pi$. It was proved by Boutet de Monvel and Sjöstrand [BS] (see also the Appendix to $[\mathrm{BG}]$ ) that $\Pi$ is a complex Fourier integral operator of positive type,

$$
\Pi \in I_{c}^{0}(X \times X, \mathcal{C})
$$

associated to a positive canonical relation $\mathcal{C}$. For definitions and notation concerning complex FIO's we refer to [MS, BS, BG]. The real points of $\mathcal{C}$ form the diagonal $\Delta_{\Sigma \times \Sigma}$ in the square of the symplectic cone

$$
\Sigma=\left\{r \alpha_{x}: r>0, x \in X\right\}
$$

where $\alpha$ is the connection form. We refer to [BG] (see Lemma 4.5 of the Appendix). Moreover, in [BS] a parametrix is constructed for $\Pi$, from which it follows (see [Ze]) that

$$
\Pi_{N}(x, y) \sim N \int_{0}^{\infty} \int_{0}^{2 \pi} e^{N\left(-i \theta+t \psi\left(r_{\theta} x, y\right)\right)} s\left(r_{\theta} x, y, N t\right) d \theta d t,
$$

where $s(x, y, t) \sim \sum_{k=0}^{\infty} t^{m-k} s_{k}(x, y) \in S^{m}\left(X \times X \times \mathbb{R}_{+}\right)$is a classical symbol of order $m$. Here, ' $\sim$ ' means modulo a rapidly decaying term (i.e., a term whose $\mathcal{C}^{j}$ norms are $O\left(N^{-k}\right)$ for all $j, k)$.

To describe the phase in (43), we let $e_{L}$ be a nonvanishing holomorphic section of $L$ over an open $U \subset M$, and consider the analytic extension' $a(z, w)$ of $a(z, z):=a(z)=\left\|e_{L}(z)\right\|_{h}^{-2}$ in $U \times U$ such that $a(w, z)=\overline{a(z, w)}$ on $U \times U$. Using coordinates $(z, \theta)$ for the point $x=e^{i \theta} a(z)^{\frac{1}{2}} e_{L}(z) \in X$, we have

$$
\psi\left(x_{1}, x_{2}\right)=-1+e^{i\left(\theta_{1}-\theta_{2}\right)} \frac{a\left(z_{1}, z_{2}\right)}{\sqrt{a\left(z_{1}\right)} \sqrt{a\left(z_{2}\right)}} .
$$

Again assuming that the metric $\omega$ on $M$ is the pull-back of $\omega_{\mathrm{FS}}$, we claim that the phase equals:

$$
\psi(x, y)=-1+\langle\iota(x), \overline{\iota(y)}\rangle,
$$

where $\iota: X \rightarrow \mathbb{C}^{2 d+1}$ is the lift of $\Phi$ given by

$$
\iota(z, \theta)=e^{i \theta}\left(\sum\left|f_{j}(z)\right|^{2}\right)^{-\frac{1}{2}}\left(f_{0}(z), \ldots, f_{d}(z)\right) .
$$

To see this, let us recall the Szegö kernel of the hyperplane section bundle $\mathcal{O}(1) \rightarrow \mathbb{C P}^{d}$ over projective space with the Fubini-Study metric. We take $U=\left\{z_{0} \neq 0\right\} \approx \mathbb{C}^{d}$, and we consider the local frame $e=z_{0}$. Using the local coordinates $\left[1, z_{1}, \ldots, z_{m}\right] \mapsto\left(z_{1}, \ldots, z_{m}\right) \in \mathbb{C}^{d}$, we then have $a^{\mathbb{C P}^{d}}(z)=\|e(z)\|^{-2}=1+\sum_{j=1}^{d}\left|z_{j}\right|^{2}$. Hence $a^{\mathbb{C P}^{d}}$ has the real-analytic extension

$$
a^{\mathbb{C P}^{d}}(z, w)=1+\sum_{j=1}^{d} z_{j} \bar{w}_{j}
$$

and (45) follows.

Remark: In the case of a toric variety $M_{P}^{c}$, (45) becomes

$$
\psi(x, y)=-1+\sum_{\alpha \in P} \widehat{m}_{\alpha}^{P}(x){\widehat{\widehat{m}_{\alpha}^{P}(y)}} .
$$


4.1. Proof of Lemma 4.2. We assume as known the following facts about complex Fourier integral operators and Toeplitz operators:

- $A \in I_{c}^{m}(X \times X, \mathcal{C})$ possesses a principal symbol $\sigma_{A}$ which is a half-density (times a Maslov factor) along the underlying canonical relation $\mathcal{C}$. (In the Toeplitz case, it is a symplectic spinor.)

- We can compose operators in $I_{c}^{m}(X \times X, \mathcal{C})$ on the left and right by elements $B \in$ $\Psi^{k}(X)$ and $\sigma_{A B}=\sigma_{A} \sigma_{B}=\sigma_{B A}$. (The same is true of Toeplitz operators.)

- If $A \in I_{c}^{m}(X \times X, \mathcal{C})$ and if $\sigma_{A}=0$, then $A \in I_{c}^{m-1}(X \times X, \mathcal{C})$ (and also for Toeplitz operators).

- $\Pi$ and $\Pi_{\iota}$ are elliptic in that their symbols are nowhere vanishing. See [BS] for the symbol in the complex FIO sense and [BG, §11] for the symbol in the Toeplitz sense.

We now give the proof:

Proof. As mentioned above (41), $\Pi$ is a complex Fourier integral operator associated to a positive canonical relation $C$, whose real points form the isotropic relation $\Delta_{\Sigma \times \Sigma} \subset T^{*} X \times$ $T^{*} X$ (the diagonal). We observe that also $\Pi_{\iota} \in I^{*}(X \times X, \mathcal{C})$. This follows immediately from the fact, show by (45), that $\Pi$ and $\Pi_{\iota}$ are complex Fourier integral distributions with precisely the same phase functions. Since the underlying canonical relation is parametrized by the phase, they both belong to the same class of Fourier integral distributions.

Now, the principal symbol $\sigma_{\Pi}$ of $\Pi$, viewed as a complex Fourier integral distribution, is a nowhere vanishing 1/2-density on $\mathcal{C}$ which is computed in [BS, Prop. 4.8]. Alternatively, viewed as a Toeplitz operator in the sense of BG (see Chapter 11), its symbol is an idempotent symplectic spinor . Similarly, the principal symbol $\sigma_{\Pi_{\iota}}$ of $\Pi_{\iota}$ is the pull back under $\iota$ of the nowhere vanishing symbol of $\Pi^{\mathbb{C P}^{d}}$.

By our normalization, $\Pi_{\iota}$ has order $-m$ (since its amplitude is a constant independent of $N)$. We therefore begin by seeking $A_{0} \in \Psi^{m}(X)$ such that $\left[A_{0}, D_{\theta}\right]=0$ and such that $\Pi-\Pi A_{0} \Pi_{\iota}$ is of order -1 . We first find $a_{0} \in C^{\infty}(M)$ such that $\sigma_{\Pi}=a_{0} \sigma_{\Pi_{\iota}}$ and choose $A_{0}$ so that $\left[A_{0}, D_{\theta}\right]=0$ and so that $\sigma_{A_{0}}=a_{0}$. Existence of such an $A_{0}$ follows by ellipticity of $\Pi_{\iota}$ and by averaging; see also [BG, Prop. 2.13]. Thus, the principal symbol of order 0 of $\Pi-\Pi A_{0} \Pi_{\iota}$ equals zero, i.e. $\Pi-\Pi A_{0} \Pi_{\iota} \in I^{-1}(X \times X, \mathcal{C})$. We denote its principal symbol by $\sigma_{-1}$. We then seek $A_{-1} \in \Psi^{m}(X)$ so that $\left[A_{-1}, D_{\theta}\right]=0$, and so that $\Pi-\Pi A_{0} \Pi_{\iota}-\Pi A_{-1} D_{\theta}^{-1} \Pi_{\iota} \in$ $I^{-2}(X \times X, \mathcal{C})$. Here, we note that $\Pi D_{\theta} \Pi$ is an elliptic Toeplitz operator; since $\Pi_{\iota}=\Pi_{\iota}$, the expressions $D_{\theta}^{-1} \Pi_{\iota}$ are well-defined. It suffices to choose $a_{-1}=\sigma_{A_{-1}} \in C^{\infty}(M)$ so that $a_{-1} \sigma_{\Pi_{\iota}}=\sigma_{-1}$. We continue in this way to obtain $a_{-j} \in C^{\infty}(M)$ always using that $\sigma_{\Pi_{\iota}}$ is nowhere vanishing. By a Borel summation argument, we can find $A \in \Psi^{m}(X)$ with the above commutation properties so that $\Pi A \Pi-\sum_{j=0}^{M} \Pi A_{-j} D_{\theta}^{-j} \Pi$ is a Toeplitz operator of order $-M-1$. Then

$$
\Pi-\Pi A \Pi_{\iota} \in I^{-\infty}(X \times X, \mathcal{C}) .
$$

We remark that an alternative to the observation that the two complex FIO's have the same phase function is that, by the choice of the Kähler form $\iota^{*} \omega_{\mathrm{FS}}, \iota$ is a symplectic (as well as holomorphic) embedding $M \subset \mathbb{C P}^{d}$. Hence, the pull back operator $\iota^{*}$ carries the class of FIO's in the class of $\Pi^{\mathbb{C P}^{d}}$ to those in the class of $\Pi$. 
4.2. Proof of Proposition 4.1. To prove the proposition, we need to relate the complex FIO $\Pi A \Pi_{\iota}$ to semi-classical Toeplitz operators. We will define the necessary terms as the proof proceeds.

We will need the following asymptotic formula for symbols. In the language of BerezinToeplitz operators, it amounts to computing the Berezin transform between covariant and contravariant symbols of a Toeplitz operator.

LEMmA 4.3. Let $\sigma_{N} \sim N^{k} \sum_{j=0}^{\infty} s_{-j} N^{-j}$ be a semiclassical symbol of order $k$. Then there exists a complete asymptotic expansion

$$
N^{-m}\left(\Pi_{N} \sigma_{N} \Pi_{N}\right)(z, z)=\sum_{j=0}^{l-1} b_{-j}(z) N^{k-j}+r_{N}^{l}(z) \quad(l \geq 1),
$$

where $b_{0}=s_{0}, b_{-1}=\Delta s_{0}+s_{-1}, \ldots$, and in general where $b_{-j}$ is a sum of differential operators applied to $s_{0}, s_{-1}, \ldots, s_{-j}$. Also, $\left\|D_{z}^{n} r_{N}^{l}(z)\right\| \leq C_{n l} N^{k-l}$.

Proof. Apply the method of stationary phase using (43]-(44) exactly as in the proof of [Ze, Theorem 1] (which is the case $\sigma=1$ ).

4.2.1. Proof of Proposition 4.1. By Lemma 4.2, there exists $A \in \Psi^{-m}(X)$ such that $\Pi_{\iota}=$ $\Pi A \Pi$. Since $\left[A, D_{\theta}\right]=0$, it follows that there exists a symbol $a_{N}$ with $\left\|\Pi_{1}^{N}-\Pi_{N} a_{N} \Pi_{N}\right\|_{\mathrm{HS}}=$ $O\left(N^{-\infty}\right)$. We may determine $a_{N}$ by using Lemma 4.3. Indeed, we have

$$
\Pi_{1}^{N}(z, z)=1 \sim \Pi_{N} a_{N} \Pi_{N}(z, z)
$$

modulo functions $r_{N}$ which tend to zero rapidly in $\mathcal{C}^{k}(M)$. It follows that $a_{0}=1$ (and then the rest of the coefficients may be determined recursively, e.g. $a_{-1}=0, a_{-2}=-D_{2} a_{0}$, and so on).

4.2.2. Alternate proof of Proposition 4.1. One could avoid using the calculus of complex Fourier integral operators or Toeplitz operators in the proof of Proposition 5.2 by further developing the calculus of semi-classical Toeplitz operators directly from the Boutet de MonvelSjöstrand parametrix as follows:

We first simplify the expression (43) by using the complex method of stationary phase to eliminate the integrals in the parametrix. The critical point set of the phase $\Phi(\theta, \lambda ; x, y):=$ $-i \theta+\lambda \psi\left(r_{\theta} x, y\right)$ is given by

$$
\left\{\begin{array}{l}
d_{\lambda} \Phi(\theta, \lambda ; x, y)=\psi\left(r_{\theta} x, y\right)=0 \Longleftrightarrow e^{i \theta}\langle\iota(x), \iota(y)\rangle=1, \\
d_{\theta} \Phi(\theta, \lambda ; x, y)=-i+d_{\theta} \lambda \psi\left(r_{\theta} x, y\right)=0 \Longleftrightarrow \lambda e^{i \theta}\langle\iota(x), \iota(y)\rangle=1 .
\end{array}\right.
$$

It is easy to see by the Schwartz inequality that a real critical point exists if and only if $x=y$, in which case $\theta=0, \lambda=1$ and we obtain the familiar expansion along the diagonal. When $x \neq y$ we deform the contour to $|\zeta|=e^{\tau}\left(\zeta=e^{i \theta+\tau}\right)$ so that $e^{i \theta+\tau}\langle\iota(x), \iota(y)\rangle=1$. This is possible as long as $\langle\iota(x), \iota(y)\rangle \neq 0$, as happens near the diagonal, where the parametrix is valid. Because the phase is linear in $\lambda$ it is clear that the critical point is non-degenerate if and only if $\langle\iota(x), \iota(y)\rangle \neq 0$ and that the Hessian determinant equals $|\langle\iota(x), \iota(y)\rangle|^{2}$. On the critical set the phase equals $-i \theta+\tau=\log \langle\iota(x), \iota(y)\rangle$, hence we have

$$
\Pi_{N}(x, y)=e^{N \log \langle\iota(x), \iota(y)\rangle} S_{N}(x, y)+W_{N}(x, y),
$$


where $S_{N}(x, y) \sim \sum_{k=0}^{\infty} N^{m-k} S_{k}(x, y)$, and where $W_{N}(x, y)$ is a smooth uniformly rapidly decaying function. Here, we have absorbed the remainder in the parametrix construction as well as the remainder in the stationary phase expansion of the parametrix in $W_{N}$. Note that the first term may be smaller than the second outside a tubular neighborhood of radius $N^{-1 / 2}$ of the diagonal.

As above, we use Lemma 4.3 to find a symbol $a_{N}$ so that (47) holds and hence the kernels $\Pi_{1}^{N}$ and $\Pi_{N} a_{N} \Pi_{N}$ agree on the diagonal modulo smoothing symbols. Next, we note that both $\Pi_{1}^{N}(x, y)$ and $\Pi_{N} a_{N} \Pi_{N}(x, y)$ are complex oscillatory functions with common phase

$$
\Psi(z, w)=\log \langle\iota(x), \iota(y)\rangle .
$$

In the case of $\Pi_{1}^{N}$, this follows from (38). Indeed, we simply have:

$$
\Pi_{1}^{N}(x, y)=e^{N \log \langle\iota(x), \iota(y)\rangle} .
$$

In the case of $\Pi_{N} a_{N} \Pi_{N}$, we apply the method of complex stationary phase to the integral formula

$$
\Pi_{N} a_{N} \Pi_{N}(x, y) \sim \int_{M_{P}} e^{N \Psi(u ; x, y)} S_{N}(x, u) a_{N}(u) S_{N}(u, y) d V(u),
$$

coming from (49), with

$$
\Psi(u ; x, y)=\langle\iota(x), \iota(u)\rangle+\langle\iota(u), \iota(y)\rangle .
$$

It follows that there exists an amplitude $A_{N}$ defined near the diagonal such that

$$
\Pi_{N} a_{N} \Pi_{N}(x, y)=A_{N}(x, y) e^{N \log \langle\iota(x), \iota(u)\rangle}+V_{N}(x, y),
$$

where $V_{N}$ is a new smoothing operator.

Recalling that $X \subset L^{*}$, we extend $A_{N}$ to $L^{*} \times L^{*}$ so that it is of the form

$$
A_{N}\left(z, \lambda ; w, \lambda^{\prime}\right)=\left(\lambda \bar{\lambda}^{\prime}\right)^{N} \tilde{A}_{N}(z, w),
$$

where we use a local holomorphic frame to write $x=(z, \lambda), y=\left(w, \lambda^{\prime}\right) \in L^{*}$. We note that $\tilde{A}_{N}(z, w)$ is holomorphic in $z$ and anti-holomorphic in $w$ near the diagonal. To see this, we first conclude from the construction in $[\mathrm{BS}]$ using the $*$-product that the symbol $s(x, y, t) \sim \sum_{k=0}^{\infty} t^{m-k} s_{k}(x, y)$ in (43) extends to a symbol on $L^{*} \times L^{*}$ that is holomorphic in $x$ and anti-holomorphic in $y$. It follows by the stationary phase method described above that the same is true for the symbol $S_{N}(x, y)$ in (49), and then by (153) that the same is also true for $A_{N}$ as claimed. (Note that in terms of coordinates $(z, \theta)$ on $X$, the function $z \mapsto A_{N}\left(z, \theta ; w, \theta^{\prime}\right)=e^{i N\left(\theta-\theta^{\prime}\right)} \sqrt{a(z) a(w)} \tilde{A}_{N}(z, w)$ is not holomorphic in $z$.)

The amplitude of $\Pi_{1}^{N}-\Pi_{N} a_{N} \Pi_{N}$ becomes $\left(\lambda \bar{\lambda}^{\prime}\right)^{N}\left(1-\tilde{A}_{N}(z, w)\right)$, where we can write $1-\tilde{A}_{N} \sim B_{0}+B_{1} N^{-1}+B_{2} N^{-2}+\cdots$. Since $\Pi_{1}^{N}-\Pi_{N} a_{N} \Pi_{N}(z, z)=O\left(N^{-\infty}\right)$ by our choice of $a_{N}$ above, it follows that $B_{j}(z, z)=0$ (for all $j$ ). Since $B_{j}(z, w)$ vanishes on the diagonal and is holomorphic in $z$ and anti-holomorphic in $w$, it must be identically 0 . Hence, $\Pi_{1}^{N}-\Pi_{N} a_{N} \Pi_{N}(x, y)$ is a smoothing operator.

\section{SZEGÖ KERNELS ON TORIC VARIETIES}

The purpose of this section is to give a special construction of the Szegö kernels

$$
\Pi_{N}^{M_{P}}(x, y)=\sum_{\alpha \in N P} \frac{1}{\left\|\chi_{\alpha}^{P}\right\|_{M_{P}}^{2}} \chi_{\alpha}^{P}(x) \overline{\chi_{\alpha}^{P}(y)},
$$


of a toric variety. The first step, Lemma [5.1, is to give an exact formula for $\Pi_{N}^{M_{P}}$ as the composition of a certain Toeplitz-Fourier multiplier denoted $(\mathcal{P Q})^{-1}$ and the pull back of the Fubini-Study kernel under a monomial embedding. The pulled-back Fubini-Study kernel is very simple to analyze and is the raison d'etre of our method. The Toeplitz-Fourier multiplier requires more work. In the second step, Lemma [5.2, we prove that this operator is a $\mathbf{T}^{m+1}$-invariant Toeplitz operator of order $m$ on $X_{P}$ (modulo a smoothing operator).

5.1. Proof of Theorem 1.1: The exact formula. Our exact formula for the Szegö kernel of a toric variety involves two ingredients: The first is the kernel $\Pi_{1}^{N}$ given by (38)-(39)). The second ingredient is the Fourier multiplier $\mathcal{M}$ defined by the eigenvalues (91). We lift the multiplier to $X$ as follows: We observe that each sequence of functions can be re-defined as a single function on the homogenized lattice cone $\Lambda_{P}=\bigcup_{N=1}^{\infty} \widehat{N P}$ defined in Section 3 , We define $\mathcal{P}, \mathcal{Q}: \Lambda_{P} \rightarrow \mathbb{R}_{+}$by

$$
\begin{aligned}
\mathcal{P}\left(\widehat{\alpha}^{N}\right):=\mathcal{P}_{N}(\alpha) & =\#\left\{\left(\beta_{1}, \ldots, \beta_{N}\right): \beta_{j} \in P, \beta_{1}+\cdots+\beta_{N}=\alpha\right\}, \\
\mathcal{Q}\left(\widehat{\alpha}^{N}\right):=\mathcal{Q}_{N}(\alpha) & =\int_{X_{P}}\left|\widehat{\chi}_{\alpha}^{P}(x)\right|^{2} d \operatorname{Vol}_{X_{P}}(x),
\end{aligned}
$$

and define Toeplitz Fourier multipliers:

$$
\begin{aligned}
& \mathcal{P}(D) \widehat{\chi}_{\alpha}^{P}=\mathcal{P}\left(\widehat{\alpha}^{N}\right) \widehat{\chi}_{\alpha}^{P}, \quad \mathcal{Q}(D) \widehat{\chi}_{\alpha}^{P}=\mathcal{Q}\left(\widehat{\alpha}^{N}\right) \widehat{\chi}_{\alpha}^{P}, \quad \alpha \in N P \\
& \mathcal{M}:=(\mathcal{P} \mathcal{Q})^{-1} .
\end{aligned}
$$

(Recall that $\mathcal{P}_{N}(\alpha) \geq 1$ by (13) , and hence $\mathcal{P} \mathcal{Q}\left(\widehat{\alpha}_{N}\right)>0$.) We write $\mathcal{M}_{N}$ for the restriction of $\mathcal{M}$ to functions in the $N$-th subspace.

We now specialize the kernels (38)-(39) to the case $M=M_{P}$ :

$$
\widetilde{\Pi}_{1}^{M_{P}}(x, y)=\left\langle\iota_{P}(x), \overline{\iota_{P}(y)}\right\rangle=\sum_{\alpha \in P} \widehat{m}_{\alpha}^{P}(x) \overline{\widehat{m}_{\alpha}^{P}(y)}, \quad \Pi_{1}^{N}(x, y)=\left[\widetilde{\Pi}_{1}^{M_{P}}(x, y)\right]^{N},
$$

where $\iota_{P}: X_{P} \rightarrow S^{2 d+1}$ is the lift of the monomial embedding $M_{P} \hookrightarrow \mathbb{C P}^{d}$, as described in \$2.2. We shall choose the constants $c_{\alpha}=1$ for all $\alpha \in P$, so that $\widehat{m}_{\alpha}^{P}=\widehat{\chi}_{\alpha}^{P}$. (Except for Lemma 5.1 below, all our results hold without change for arbitrary $\left\{c_{\alpha}\right\}$.)

We now obtain the exact formula for the Szegö kernel in Theorem 1.1. The proof is very simple.

LemMa 5.1. The Szegö kernels of a toric variety $M_{P}$ factor as follows:

$$
\Pi_{N}^{M_{P}}=\mathcal{M}_{N} \circ \Pi_{1}^{N}
$$

Proof. First, we have by definition,

$$
\Pi_{N}^{M_{P}}(x, y)=\sum_{\alpha \in N P} \frac{1}{\mathcal{Q}_{N}(\alpha)} \widehat{\chi}_{\alpha}^{P}(x) \overline{\widehat{\chi}_{\alpha}^{P}(y)} .
$$

On the other hand, by definition of the partition function, we also have

$$
\Pi_{1}^{N}(x, y)=\sum_{\alpha \in N P} \mathcal{P}_{N}(\alpha) \widehat{\chi}_{\alpha}^{P}(x) \overline{\widehat{\chi}_{\alpha}^{P}(y)}
$$


We note that the $N$-th power of $\Pi_{1}^{M_{P}}$ gives the sum over the correct set of exponents but does not have the correct normalizing coefficients. We need to divide each term by $\mathcal{P}_{N}(\alpha) \mathcal{Q}_{N}(\alpha)$ to adjust the coefficients. That is just what the lemma claims.

Of course,

$$
(\mathcal{P} \mathcal{Q})^{-1} \Pi_{1}^{N}=\Pi_{N}^{M_{P}}(\mathcal{P} \mathcal{Q})^{-1} \Pi_{N}^{M_{P}} \Pi_{1}^{N}
$$

so $\mathcal{M}$ need only be defined on $\mathcal{H}^{2}$.

5.2. Proof of Theorem 1.1: $\mathcal{M}$ is a Toeplitz operator. The explicit formula of Lemma 5.1 is difficult to use without a detailed analysis of the multiplier $\mathcal{M}$. In this section, we prove that $\mathcal{M}$ is a Toeplitz operator, i.e. possesses a symbol. This has numerous implication for $\mathcal{M}$, in particular, that it has polyhomogeneous expansions along rays.

We begin with some background on symbols. We consider symbols $\sigma$ of the form $\sigma\left(z, D_{\theta}\right)$, where $\sigma(z, N)$ is a semiclassical symbol. Here, we say $\sigma \in S^{k}(M \times \mathbb{N})$ is a semiclassical symbol of order $k$ if

$$
\sigma_{N}(z)=\sigma(z, N)=N^{k} \sum_{j=0}^{l-1} a_{j}(z) N^{-j}+r_{N}^{l}(z), \quad \text { with }\left\|D_{z}^{n} r_{N}^{l}(z)\right\| \leq C_{n l} N^{k-l} \quad(l \geq 1)
$$

where $a_{j} \in \mathcal{C}^{\infty}(M)$. It is a smoothing symbol if $\left\|D_{z}^{n} \sigma_{N}(z)\right\| \leq C_{n} N^{-l}$ for all $n, l$. The Toeplitz operator associated to a symbol $\sigma$ is the operator $\Pi \sigma\left(z, D_{\theta}\right) \Pi$, where $D_{\theta}$ denotes the symbol of $\frac{\partial}{\partial \theta}$ (the $S^{1}$ generator). Its symbol is the polyhomogeneous function on the symplectic cone $\Sigma=\left\{\left(x, r \alpha_{x}\right): r>0\right\} \subset T^{*} X$ given by

$$
\sigma\left(z, p_{\theta}\right) \sim \sum_{j=0}^{\infty} a_{j}(z) p_{\theta}^{k-j}
$$

Since $\sigma$ commutes with the $S^{1}$ action, we have $\Pi \sigma \Pi=\sum_{N} \Pi_{N} \sigma_{N} \Pi_{N}$.

The goal of this section is to prove that $\Pi_{N}(\mathcal{P} \mathcal{Q})^{-1} \Pi_{N}$ is a semi-classical Toeplitz operator in the following sense:

Lemma 5.2. There exists a $\mathbf{T}^{m}$-invariant symbol $\sigma\left(z, D_{\theta}\right)$ so that $\mathcal{M}=\Pi \sigma\left(z, D_{\theta}\right) \Pi$. Equivalently, there exists a symbol $\sigma_{N}$ of order $m$ with principal symbol equal to 1 and a smoothing operator $R_{N}$ so that

$$
\Pi_{N}(\mathcal{P} \mathcal{Q})^{-1} \Pi_{N}=\Pi_{N} \sigma_{N} \Pi_{N}+R_{N}
$$

Proof. In the toric case, $\Pi_{N}(\mathcal{P} \mathcal{Q})^{-1} \Pi_{N}$ is the inverse of $\Pi_{N}(\mathcal{P} \mathcal{Q}) \Pi_{N}=\Pi_{1}^{N}$ on $\mathcal{H}^{2}\left(X_{P}\right)$. From Lemma 4.1 we may write $\Pi_{N}(\mathcal{P} \mathcal{Q}) \Pi_{N} \sim \Pi_{N} a_{N} \Pi_{N}$ modulo smoothing operators.

We note that we may invert $\Pi a \Pi$ in the class of Toeplitz operators; i.e., there exists a symbol $\sigma_{N}$ such that

$$
\Pi_{N} \sigma_{N} \Pi_{N} \circ \Pi_{N} a_{N} \Pi_{N} \sim \Pi_{N}
$$

modulo smoothing operators. Such an inverse symbol exists since $a_{0}=1$. The algebraic formalism in which the inverse is calculated is that of $*$-products of semiclassical symbols. We recall that composition of Toeplitz operators defines a $*$-product on semiclassical symbols by the formula

$$
\Pi_{N} a_{N} \Pi_{N} \circ \Pi_{N} b_{N} \Pi_{N} \sim \Pi_{N} a_{N} * b_{N} \Pi_{N} .
$$

The formula for $*$ may be worked out directly from the parametrix (43) and the inverse can be computed from this formula (see [Gu2, BS]). 
By (57) we obtain a symbol $\sigma_{N}$ (with principal symbol equal to 1 ) such that

$$
\Pi_{N} \sigma_{N} \Pi_{N} \Pi_{N}(\mathcal{P Q}) \Pi_{N} \sim \Pi_{N} .
$$

Multiplying both sides by $\Pi_{N}(\mathcal{P Q})^{-1} \Pi_{N}$, we conclude the proof.

Remark: We emphasize that the distinguishing features of the toric case in Proposition 4.1 and Lemma 5.2 are the exact factorization and the fact that the operator $\Pi(\mathcal{P} \mathcal{Q}) \Pi$ mediating between $\Pi$ and $\Pi_{\iota}$ is invertible. This is due to the fact that sections of $L$ generate the ring $\oplus_{N=1}^{\infty} H^{0}\left(M, L^{N}\right)$ in the toric case. In general they do not and the exact representation $\Pi=B \Pi_{\iota}$ in the toric case is only valid modulo smoothing operators.

5.3. Polytope characters. As a corollary of Lemma 5.2, we obtain the following formula for the character of the torus action:

Proposition 5.3. There exists a symbol $\sigma_{N} \in S^{m}(M, \mathbb{N})$ with principal symbol equal to 1 and a smoothing operator $R_{N}$ such that for $t \in \mathbf{T}^{m}$, we have

$$
\begin{aligned}
\chi_{N P}(t)= & \int_{X_{P}} \sigma_{N}(x) \Pi_{1}^{N}(t \cdot x, x) d \operatorname{Vol}_{X_{P}}(x) \\
& +\int_{X_{P}} \int_{X_{P}} R_{N}(y, x) \Pi_{1}^{N}(t \cdot x, y) d \operatorname{Vol}_{X_{P}}(x) d \operatorname{Vol}_{X_{P}}(y) .
\end{aligned}
$$

Proof. We shall use the elementary formula for the polytope character:

$$
\chi_{N P}\left(e^{i \varphi}\right)=\int_{M_{P}} \Pi_{N}\left(e^{i \varphi} \cdot x, x\right) d V(x) .
$$

Applying $\Pi_{1}^{N}=\Pi_{N}(\mathcal{P} \mathcal{Q}) \Pi_{N}$ to the identity of Lemma 5.2, we obtain

$$
\Pi_{N}=\left(\Pi_{N} \sigma_{N} \Pi_{N}+R_{N}\right) \Pi_{1}^{N} .
$$

Now let $T_{t}$ denote the translation operator $f(x) \mapsto f(t \cdot x)$ on $\mathcal{L}^{2}\left(X_{P}\right)$. Since $\left[T_{t}, \Pi_{N}\right]=0$, we then have

$$
T_{t} \Pi_{N}=T_{t} \Pi_{N}\left(\sigma_{N}+R_{N}\right) \Pi_{1}^{N}=\Pi_{N} T_{t}\left(\sigma_{N}+R_{N}\right) \Pi_{1}^{N} .
$$

Therefore,

$$
\begin{aligned}
\int_{X_{P}} \Pi_{N}(t \cdot x, x) d \operatorname{Vol}_{X_{P}}(x)=\operatorname{Trace} T_{t} \Pi_{N}=\operatorname{Trace} \Pi_{N} T_{t}\left(\sigma_{N}+R_{N}\right) \Pi_{1}^{N} \\
=\operatorname{Trace} T_{t} \sigma_{N} \Pi_{1}^{N}+\operatorname{Trace} T_{t} R_{N} \Pi_{1}^{N} \\
=\int_{X_{P}} \sigma_{N}(t \cdot x) \Pi_{1}^{N}(t \cdot x, x) d \operatorname{Vol}_{X_{P}}(x) \\
\quad+\int_{X_{P}} \int_{X_{P}} R_{N}(t \cdot x, y) \Pi_{1}^{N}(y, x) d \operatorname{Vol}_{X_{P}}(x) d \operatorname{Vol}_{X_{P}}(y) .
\end{aligned}
$$

Making the change of variables $x \mapsto t^{-1} \cdot x$, we then obtain the desired formula from (59). 


\section{REFERENCES}

[BG] L. Boutet de Monvel and V. Guillemin, The spectral theory of Toeplitz operators, Annals of Mathematics Studies 99, Princeton University Press, Princeton, NJ, 1981.

[BS] L. Boutet de Monvel and J. Sjöstrand, Sur la singularité des noyaux de Bergman et de Szegö, Asterisque 34-35 (1976), 123-164.

[De] T. Delzant, Hamiltoniens périodiques et image convexe de l'application moment, Bull. Soc. Math. France 116 (1988), 315-339.

[Fu] W. Fulton, Introduction to Toric Varieties, Annals of Mathematics Studies 131, Princeton Univ. Press, Princeton, NJ, 1993.

[GKZ] I. M. Gelfand, M. M. Kapranov and A. V. Zelevinsky, Discriminants, resultants, and multidimensional determinants, Mathematics: Theory and Applications, Birkhäuser, Boston, 1994.

[Gu1] V. Guillemin, Moment Maps and Combinatorial Invariants of Hamiltonian $T^{n}$-Spaces, Progress in Math. 122, Birkhäuser, Boston, 1994.

[Gu2] V. Guillemin, Star products on compact pre-quantizable symplectic manifolds. Lett. Math. Phys. 35 (1995), 85-89.

[Hö] L. Hörmander, The Analysis of Linear Partial Differential Operators, I (Second Ed.) Springer, Berlin, 1990.

[LT] P. Li and G. Tian, On the heat kernel of the Bergmann metric on algebraic varieties, J. Amer. Math. Soc. 8 (1995), 857-877.

[MS] A. Melin and J. Sjöstrand, Fourier integral operators with complex-valued phase functions, Fourier integral operators and partial differential equations (Colloq. Internat., Univ. Nice, Nice, 1974), Lecture Notes in Math., Vol. 459, Springer, Berlin, 1975, pp. 120-223.

[STZ1] B. Shiffman, T. Tate and S. Zelditch, Distribution laws for integrable eigenfunctions (in preparation).

[STZ2] B. Shiffman, T. Tate and S. Zelditch, Toric varieties and lattice path combinatorics (in preparation).

[SZ1] B. Shiffman and S. Zelditch, Distribution of zeros of random and quantum chaotic sections of positive line bundles, Comm. Math. Phys. 200 (1999), 661-683.

[SZ2] B. Shiffman and S. Zelditch, Random polynomials with prescribed Newton polytope, preprint 2002 (arXiv:math.AG/0211391).

[Ze] S. Zelditch, Szegö kernels and a theorem of Tian, IMRN 6 (1998), 317-331.

Department of Mathematics, Johns Hopkins University, Baltimore, MD 21218, USA

E-mail address: shiffman@math.jhu.edu

Department of Mathematics, Keio University, Keio University 3-14-1 Hiyoshi KohokU-Ku, YOKOHAMA, 223-8522 JAPAN

E-mail address: tate@math.keio.ac.jp

Department of Mathematics, Johns Hopkins University, Baltimore, MD 21218, USA

E-mail address: zelditch@math.jhu.edu 